\title{
No-Go Theorem of Leibniz Rule and Supersymmetry on the Lattice
}

\section{Mitsuhiro Kato}

Institute of Physics, University of Tokyo, Komaba, Meguro-ku, Tokyo 153-8902, Japan

E-mail: kato@hep1.c.u-tokyo.ac.jp

\section{Makoto Sakamoto*i}

Department of Physics, Kobe University, Nada-ku, Hyogo 657-8501, Japan

E-mail: dragonakobe-u.ac.jp

\section{Hiroto So}

Department of Physics, Ehime University, Bunkyou-chou 2-5, Matsuyama 790-8577, Japan E-mail: so@phys.sci.ehime-u.ac.jp

An obstacle to realize supersymmetry on a lattice is the breakdown of Leibniz rule. We give a proof of a no-go theorem that it is impossible to construct a lattice field theory in an infinite lattice volume with any nontrivial field products and difference operators that satisfy the following three properties: (i) translation invariance, (ii) locality and (iii) Leibniz rule. We then propose a way to escape from the no-go theorem by introducing infinite flavors, and present a lattice model of $N=2$ supersymmetric quantum mechanics equipped with the full exact supersymmetry.

The XXVI International Symposium on Lattice Field Theory

July 14-19 2008

Williamsburg, Virginia, USA

\footnotetext{
*speaker

$\dagger$ The authors would like to thank to K. Nagata and N. Ukita for useful discussions. This work is supported in part by the Grant-in-Aid for Scientific Research (No.19540272 (M.K.), No.18540275 (M.S.) and No.17540242, No.20540274 (H.S.)) by the Japanese Ministry of Education, Science, Sports and Culture.
} 


\section{Introduction}

Over the last thirty years, a considerable number of attempts have been made to construct lattice supersymmetric models [1, 2, 3]. However, none of them have been fully successful. To see an obstacle in realizing supersymmetry on a lattice, let us consider supersymmetry invariance in the continuum, which may be best seen in the superfield formulation. We first note that an infinitesimal supersymmetry transformation for any superfield $X(x, \theta)$ is essentially written in the form $\delta_{\varepsilon} X(x, \theta)=\varepsilon\left(\partial_{\theta}+\theta \partial_{x}\right) X(x, \theta)$, where $\partial_{x}$ and $\partial_{\theta}$ denote the derivatives with respect to the spacetime coordinate $x$ and the grassmann coordinate $\theta$, respectively, and $\varepsilon$ is an infinitesimal grassmann parameter. Then, the action invariance under the transformation can easily be shown as

$$
\begin{aligned}
S\left(\Phi+\delta_{\varepsilon} \Phi\right)-S(\Phi) & \equiv \int d x \int d \theta\left\{\mathscr{L}\left(\Phi(x, \theta)+\delta_{\varepsilon} \Phi(x, \theta)\right)-\mathscr{L}(\Phi(x, \theta))\right\} \\
& =\int d x \int d \theta \delta_{\varepsilon} \mathscr{L}(x, \theta) \\
& =0,
\end{aligned}
$$

where $\Phi$ stands for a superfield. The last equality immediately follows from the fact that $\int d x \partial_{x}=0$ and $\int d \theta \partial_{\theta}=0$.

In lattice formulation, $x, \partial_{x}$ and $\int d x$ should be replaced by $n, \nabla$ and $\sum_{n}$, respectively, where $n$ and $\nabla$ denote a position and a difference operator on a lattice. Since ordinary difference operators satisfy the relation $\sum_{n} \nabla=0$, most of the properties in the continuum turn out to hold even on a lattice. A nontrivial step, however, exists. To go from the first line to the second in eq. 11.1), we need a Leibniz rule for the supersymmetry transformations, i.e. $\delta_{\varepsilon}(\Phi \Psi)=\left(\delta_{\varepsilon} \Phi\right) \Psi+\Phi\left(\delta_{\varepsilon} \Psi\right)$. The Leibniz rule is trivially satisfied in the continuum but not on a lattice. This is because the supersymmetry transformations include spacetime derivatives, which should be replaced by some difference operators on a lattice, and naive difference operators are known to violate the Leibniz rule [4, 5, 6, 7. Thus, it will be important to clarify the question whether the Leibniz rule can be realized on a lattice.

Our answer is negative. In the next section, we give a proof of the no-go theorem [8]:

No-Go Theorem. It is impossible to construct a lattice field theory in an infinite lattice volume with any nontrivial field products and difference operators that satisfy the following three properties: (i) translation invariance, (ii) locality and (iii) Leibniz rule.

We, however, propose a way to escape from the no-go theorem and present a lattice formulation equipped with a Leibniz rule in Section 3. Then, we explicitly construct a lattice model of $N=2$ superesymmetric quantum mechanics and discuss the full exact supersymmetric properties from various points of view in Section 4. Section 5 is devoted to summary and discussions.

\section{A Proof of the No-Go Theorem}

In this section, we present a proof of the no-go theorem stated in the Introduction. In order to analyze a Leibniz rule on a lattice, we must generalize a product between fields and a difference operator on a field. A lattice field product between $\phi_{n}$ and $\psi_{n}$ and a difference operator on a field $\phi_{n}$ are defined, respectively, as 


$$
(\phi \cdot \psi)_{n} \equiv \sum_{l, m} C_{l m n} \phi_{l} \psi_{m}, \quad(D \phi)_{n} \equiv \sum_{m} D_{m n} \phi_{m},
$$

where the indices $l, m, n$ denote positions on a lattice of an infinite size.

The coefficients $C_{l m n}$ and $D_{m n}$ cannot be arbitrary. The difference operator for a constant field should vanish, i.e. $\sum_{m} D_{m n}=0$. We require that they are translationally invariant, such that $C_{l m n}=C(l-n, m-n)$ and $D_{m n}=D(m-n)$. We further require the field product and the difference operator to be local. The locality property is important in constructing local field theories after the continuum limit. To make the locality manifest, we define Fourier transform of the coefficients $C(k, l)$ and $D(m)$ by

$$
\hat{C}(v, w) \equiv \sum_{k, l} C(k, l) v^{k} w^{l}, \quad \hat{D}(z) \equiv \sum_{m} D(m) z^{m},
$$

where $v, w, z$ are $S^{1}$-variables given by $v=e^{i p}, w=e^{i q}, z=e^{i r}(0 \leq p, q, r<2 \pi)$. We note that the condition $\sum_{m} D_{m n}=0$ can be replaced by an "initial" condition

$$
\hat{D}(1)=0 \text {. }
$$

A crucial observation to prove the no-go theorem is that the locality of the field product and the difference operator allows $v, w, z$ to extend to an annulus domain $1-\varepsilon<|v|,|w|,|z|<1+\varepsilon$ with a positive constant $\varepsilon$ and then $\hat{C}(v, w)$ and $\hat{D}(z)$ can be regarded as holomorphic functions on the annulus domain [8].

We are now ready to present a proof of the no-go theorem. The Leibniz rule $D(\phi \cdot \psi)=$ $(D \phi) \cdot \psi+\phi \cdot(D \psi)$ can be translated into a relation between the field product and the difference operator as

$$
\sum_{k} C_{l m k} D_{k n}=\sum_{k} C_{k m n} D_{l k}+\sum_{k} C_{l k n} D_{m k} .
$$

In terms of the holomorphic functions $\hat{C}(v, w)$ and $\hat{D}(z)$, the above relation can be rewritten into a simple form

It follows that

$$
\hat{C}(v, w)(\hat{D}(v w)-\hat{D}(v)-\hat{D}(w))=0 .
$$

$$
\hat{D}(v w)-\hat{D}(v)-\hat{D}(w)=0
$$

on a restricted domain with $\hat{C}(v, w) \neq 0$. We can, however, show that the relation 2.6) holds on the annulus domain without the constraint $\hat{C}(v, w) \neq 0$ by virtue of the holomorphy. To see this, we first note that since $\hat{C}(v, w)$ is holomorphic, $\hat{C}(v, w)$ can vanish at most at isolated points, otherwise $\hat{C}(v, w)$ should vanish identically due to the identity theorem on holomorphic functions. It turns out that the equation (2.6) holds on the whole annulus domain without the condition $\hat{C}(v, w) \neq 0$, again due to the identity theorem on holomorphic functions.

A general solution to eq. (2.6) is easily found to be of the form

$$
\hat{D}(w)=\beta \log w .
$$

The coefficient $\beta$, however, has to vanish in order for $\hat{D}(w)$ to be holomorphic because the logarithmic function $\log w$ is not holomorphic on the annulus domain and hence is not local. Thus, we conclude that there is no nontrivial difference operator with the properties of (i) translation invariance, (ii) locality and (iii) Leibniz rule. This completes the proof. 
We would like to make a few comments on the locality. Our proof implies that if we allow the difference operator to be non-local (but still the field product to be local), the difference operator (2.7) is a unique solution, which is known as a SLAC-type derivative [4]. On the other hand, if we allow the field product to be non-local, we can obtain local difference operators which are solutions to eq. (2.5). For example, if we take $C_{l m n}=c$ (=constant), which is highly non-local, then we find $\hat{C}(v, w)=(2 \pi i)^{2} c \delta(v-1) \delta(w-1)$. It then follows that any (local) difference operators with the property (2.3) satisfy the equation (2.5) trivially. Another example will be found in Ref.(5]

\section{Escape from the No-Go Theorem}

Although we have proved a no-go theorem about the Leibniz rule on a lattice, it is still worthwhile continuing to search for local difference operators and field products satisfying the Leibniz rule because they will make it possible to construct lattice models with the full exact supersymmetry.

A way to escape from the no-go theorem may be to introduce multi-flavors in the system. ${ }^{1} \mathrm{~A}$ field product and a difference operator are naturally extended as

$$
(\phi \cdot \psi)_{n}^{c} \equiv \sum_{l, m} \sum_{a, b=1}^{N} C_{l m n}^{a b c} \phi_{l}^{a} \psi_{m}^{b}, \quad(D \phi)_{n}^{b} \equiv \sum_{m} \sum_{a=1}^{N} D_{m n}^{a b} \phi_{m}^{a},
$$

where $a, b, c$ denote flavor indices. A Leibniz rule in multi-flavor systems can be expressed as

$$
\sum_{k} \sum_{d=1}^{N} C_{l m k}^{a b d} D_{k n}^{d c}=\sum_{k} \sum_{d=1}^{N} C_{k m n}^{d b c} D_{l k}^{a d}+\sum_{k} \sum_{d=1}^{N} C_{l k n}^{a d c} D_{m k}^{b d}
$$

The translation invariance and the locality for $C_{l m n}^{a b c}$ and $D_{m n}^{a b}$ are defined in the same way as in single flavor systems, and lead to the holomorphic functions

$$
\hat{C}^{a b c}(v, w) \equiv \sum_{l, m=-\infty}^{\infty} C^{a b c}(l, m) v^{l} w^{m}, \quad \hat{D}^{a b}(z) \equiv \sum_{m=-\infty}^{\infty} D^{a b}(m) z^{m} .
$$

One might hope that $N$-flavor systems could evade the no-go theorem proved in one-flavor systems but the theorem turns out to still hold. This is because the proof can reduce to the oneflavor case by diagonalizing $\hat{D}^{a b}(z)$ such that $\hat{D}^{a b}(z)=\delta^{a b} \hat{D}^{a}(z)$. Thus, a multi-flavor extension seems not to work to solve our problem. However, there exists a loophole to escape from the no-go theorem. A key observation is that a linear combination of an infinite number of holomorphic functions is not necessarily holomorphic. The holomorphy of $\hat{C}^{a b c}(v, w)$ and $\hat{D}^{a b}(z)$ is not necessarily preserved in diagonalizing $\hat{D}^{a b}(z)$ with infinite flavors, and hence the proof for one-flavor cannot be applied to an infinite number of flavors.

In fact, we find a solution to the equation (3.2) [8]:

$$
C_{l m n}^{a b c}=\delta_{l-n, b} \delta_{n-m, a} \delta_{a+b, c}, \quad D_{m n}^{a b}=d(a-b)\left(\delta_{m-n, a-b}-\delta_{m-n,-(a-b)}\right),
$$

where $d(a-b)$ is an arbitrary function of $a-b$. The characteristic features of the above solution are listed below: They are translationally invariant because $D_{m n}^{a b}\left(C_{l m n}^{a b c}\right)$ depends only on $m-n(l-n$ and $m-n)$. They are local because the functions

$$
\hat{C}^{a b c}(v, w)=\delta_{a+b, c} v^{b} w^{-a}, \quad \hat{D}^{a b}(z)=d(a-b)\left(z^{a-b}-z^{-(a-b)}\right)
$$

\footnotetext{
${ }^{1}$ Another approach to restore a Leibniz rule with a mild non-commutativity has been proposed in Ref. 9 .
} 
are holomorphic on the annulus domain. The expressions (3.4) imply nontrivial connections between the lattice sites and the flavor indices and the necessity of an infinite number of flavors. We could diagonalize $\hat{D}^{a b}(z)$ given in eq. (3.5) but the resulting expression turns out to become non-holomorphic. Thus, we cannot apply the previous proof in the present case, as mentioned before. The solution (3.4) is local in the space direction, as they should be, but they are, in some sense, "non-local" in the flavor direction. Thus, one might say that the non-locality problem can be resolved by transferring the non-locality in the space direction to the flavor direction.

The expressions (3.4) may become more tractable by writing them into a matrix form. A lattice field $\phi_{n}^{a}$ will be replaced by a matrix $\Phi_{i j}$ with the identification of $a=i-j$ and $n=i+j$. The difference operator $(D \phi)_{n}^{a}$ turns out to be a commutator $[d, \Phi]_{i j}$, where the matrix $d$ is given by $d_{i j} \equiv$ $d(i-j)$. The field product $(\phi \cdot \psi)_{n}^{a}$ is found to be just the product of matrices, $(\Phi \Psi)_{i j}=\sum_{k} \Phi_{i k} \Psi_{k j}$. The Leibniz rule $D(\phi \cdot \psi)=(D \phi) \cdot \psi+\phi \cdot(D \psi)$ is rather trivial in the matrix representation and is replaced simply by a trivial commutator relation $[d, \Phi \Psi]=[d, \Phi] \Psi+\Phi[d, \Psi]$. The summation over the lattice sites and the flavor indices is replaced by the trace of matrices.

\section{4. $N=2$ SUSY QM on the Lattice}

Since we have succeeded to find a local difference operator and a local field product equipped with the Leibniz rule, we can construct supersymmetric lattice models. In this section, as an example, we present a lattice model of $N=2$ supersymmetric quantum mechanics, and discuss various supersymmetric properties. The details will be reported in a forthcoming paper.

Our lattice action of $N=2$ supersymmetric quantum mechanics equipped with the tools developed in the previous section is ${ }^{2}$

$$
S=\operatorname{tr}\left[\frac{1}{2}[d, q]^{2}-\frac{1}{2} F^{2}+\bar{\psi}[d, \psi]+\lambda F q^{2}+\lambda(\bar{\psi} q \psi+\bar{\psi} \psi q)\right]
$$

where $d$ is an anti-hermitian matrix and $q, F$ are bosonic matrices and $\psi, \bar{\psi}$ are fermionic ones. We should be careful about the order of the fields because they are represented by matrices. It is easy to see that the lattice action is invariant under the supersymmetry transformations

$$
\delta q=\varepsilon \bar{\psi}-\bar{\varepsilon} \psi, \quad \delta \psi=\varepsilon(-[d, q]+F), \quad \delta \bar{\psi}=\bar{\varepsilon}([d, q]+F), \quad \delta F=\varepsilon[d, \bar{\psi}]+\bar{\varepsilon}[d, \psi],
$$

where $\varepsilon$ and $\bar{\varepsilon}$ are infinitesimal grassmann parameters (but not matrices). The invariance implies that the action possesses the full exact $N=2$ supersymmetry. This is the most important property of our model. We can rewrite the lattice action (4.1) in the superfield formulation, such that

$$
S=\int d \bar{\theta} d \theta \operatorname{tr}\left[\frac{1}{2}(\bar{D} \Phi)(D \Phi)+\frac{\lambda}{3} \Phi^{3}\right]
$$

where $\Phi$ and $D, \bar{D}$ denote the superfield and the supercovariant derivatives. Furthermore, the action can be written into a $Q$-exact form [11]

$$
S=\bar{Q} Q \operatorname{tr}\left[\frac{1}{2} \bar{\psi} \psi+\frac{\lambda}{3} q^{3}\right]=-Q \bar{Q} \operatorname{tr}\left[\frac{1}{2} \bar{\psi} \psi+\frac{\lambda}{3} q^{3}\right]
$$

\footnotetext{
${ }^{2}$ The action was originally found in Ref.[10].
} 
where $Q$ and $\bar{Q}$ denote the supercharges, which generate the supersymmetry transformations (4.2). The other interesting feature is that the action has two kinds of the Nicolai mappings [12, 13, 14] given by

$$
\xi=[d, q]+\lambda q^{2}, \quad \eta=-[d, q]+\lambda q^{2} .
$$

In terms of the two Nicolai mappings, the on-shell action can be expressed in two ways:

$$
S^{\text {on-shell }}=\frac{1}{2} \xi_{i j} \xi_{j i}+\bar{\psi}_{i j} \frac{\partial \xi_{j i}}{\partial q_{k l}} \psi_{k l}=\frac{1}{2} \eta_{i j} \eta_{j i}+\bar{\psi}_{i j} \frac{\partial \eta_{l k}}{\partial q_{i j}} \psi_{k l} .
$$

It follows from the above expressions that each of the two Nicolai mappings $\xi$ and $\eta$ are found to be connected with one of the two supercharges of the $N=2$ supersymmetry [15, 16].

We should make a comment on the doubling problem. The lattice action 4.17 unfortunately suffers from the doubling in both the spectrum of bosonic and fermionic variables. We can, however, add a supersymmetric Wilson term to the lattice action 4.1. to avoid the doubling problem.

It should be noting that the lattice action is exactly supersymmetric even for a finite size of the matrices. This does not, however, contradict the no-go theorem because the theorem has been proved only for an infinite lattice volume. In fact, the locality is obscure for a finite lattice.

\section{Summary and Discussions}

We have proved the no-go theorem that it is impossible to construct a lattice field theory in an infinite lattice volume with any field products and difference operators that satisfy the following three properties: (i) translation invariance, (ii) locality and (iii) Leibniz rule. We then proposed a way to escape from the no-go theorem by introducing infinite flavors and presented a matrix realization of the lattice formulation equipped with the Leibniz rule. In terms of the tools in the matrix realization, we constructed a lattice model of $N=2$ supersymmetric quantum mechanics and discussed various interesting properties. One of the remarkable features is that the model realizes the full exact supersymmetry but most of known supersymmetric lattice models can, in contrast, possess supersymmetry at most partially. ${ }^{3}$

Since our lattice models include an infinite number of flavors, we need to reduce the number of flavors in the continuum limit at least at low energies. An attractive idea is to use an analogy between infinite flavors of our models and Kaluza-Klein modes on extra dimensions. If we can add "KK mass" terms in our lattice actions in a supersymmetric way, only finite degrees of freedom could survive at low energies.

In the previous section, we have presented a $0+1$-dimensional supersymmetric lattice model. The extension to higher dimensions will be straightforward for Wess-Zumino type models. In fact, we have succeeded to construct lattice models of $d=2 N=2$ and $d=4 N=1$ Wess-Zumino models in our formulation. The introduction of gauge fields is, however, less trivial. Our lattice models have a genuine non-commutative nature and it seems to be natural to embed vector indices (as well as spinor indices) in matrices.

\footnotetext{
${ }^{3}$ Other lattice models that realize exact supersymmetry have been proposed in Ref. 9 . However, a breakdown of the supersymmetry at the quantum level was reported [17. Another interesting idea to construct lattice models with an exact fermionic symmetry has been presented in Ref.[18, though the relation between the fermionic symmetry and supersymmetry is unclear.
} 
An advantage of our lattice formulation is that the lattice models are exactly supersymmetric even for a finite lattice size, and hence numerical simulations can be done.

The full details of the supersymmetric lattice models given in Section 4 and further progress will be reported in a forthcoming paper.

\section{References}

[1] A. Feo, Supersymmetry on the lattice, Nucl. Phys. 119 (Proc. Suppl.) (2003) 198 [hep-lat/0210015].

[2] D.B. Kaplan, Recent developments in lattice supersymmetry, Nucl. Phys. 129 (Proc. Suppl.) (2004) 109 [hep-lat/0309099]; Supersymmetry on the lattice, Eur. Phys. J. ST. 152 (2007) 89.

[3] J. Giedt, Advances and applications of lattice supersymmetry, PoS LAT2006 (2006) 008 [hep-lat/0701006].

[4] S.D. Drell, M. Weinstein and S. Yankielowicz, Strong-coupling field theories. II. Fermions and gauge fields on a lattice, Phys. Rev. D 14 (1976) 1627.

[5] P.H. Dondi and H. Nicolai, Lattice Supersymmetry, Nuovo Cim. A 41 (1977) 1.

[6] Y. Bouguenaya and D.B. Fairlie, A finite difference scheme with a Leibniz rule, J. Phys. A 19 (1986) 1049.

[7] H. So and N. Ukita, Lattice Supersymmetry, talk in Workshop on "Supersymmetry, Chiral Symmetry and Lattice Gauge Theory" (SUCAL 99), Niigata, Japan, 17-19, November (1999).

[8] M. Kato, M. Sakamoto and H. So, Taming the Leibniz rule on the lattice, JHEP 05 (2008) 057 [arXiv.0803.3121(hep-lat)].

[9] A. d'Adda, I. Kanamori, N. Kawamoto, and K. Nagata, Twisted superspace on a lattice, Nucl. Phys B 707 (2005) 100 [hep-lat/0406029]; Exact extended supersymmetry on a lattice: Twisted $N=2$ super Yang-Mills in two dimensions, Phys. Lett. B 633 (2006) 645 [hep-lat/0507029].

[10] M. Kato, M. Sakamoto and H. So, Leibniz rule and exact supersymmetry on lattice: A Case of supersymmetrical quantum mechanics, PoS LAT2005 (2006) 274 [hep-lat/0509149].

[11] E. Witten, Topological Quantum Field Theory, Commun. Math. Phys. 117 (1988) 353.

[12] H.Nicolai, On a New Characterization of Scalar Supersymmetric Theories, Phys.Lett. B 89 (1980) 341; Supersymmetry And Functional Integration Measures, Nucl.Phys. B 176 (1980) 419.

[13] S.Cecotti and L.Girardello, Stochastic Processes in Lattice (Extended) Supersymmetry, Nucl. Phys. B 226 (1983) 417.

[14] N.Sakai and M.Sakamoto, Lattice Supersymmetry and the Nicolai Mapping, Nucl. Phys. B 229 (1983) 173.

[15] G.Parisi and N.Sourlas, Supersymmetric Field Theories and Stochastic Differential Equations, Nucl. Phys. B 206 (1982) 321.

[16] S.Catterall and S.Karamov, Exact lattice supersymmetry: The Two-dimensional N=2 Wess-Zumino mode, Phys. Rev. D 65 (2002) 094501 [hep-lat/0108024].

[17] K. Nagata, Exact Lattice Supersymmetry at Large N, [arXiv:0805.4235(hep-lat)].

[18] K. Itoh, M. Kato, H. Sawanaka, H. So and N. Ukita, Towards the superYang-Mills theory on the lattice, Prog. Theor. Phys. 108 (2002) 363 [hep-lat/0112052]; Novel Approach to Super Yang-Mills Theory on Lattice -Exact fermionic symmetry and "Ichimatsu" pattern-, JHEP 0302 (2003) 033 [hep-lat/0210049]. 